'Servicio de Laboratorios Clínicos. Facultad de Medicina. Pontificia Universidad Católica de Chile. ${ }^{2}$ Departamento de Gastroenterología. Facultad de Medicina, Pontificia Universidad Católica de Chile, Santiago, Chile. aBioquímico.

La presente publicación no contó con financiamiento externo. Los autores declaran que no existen conflictos de interés ni apoyo financiero relacionados al presente trabajo.

Recibido el 2 de septiembre de 2015, aceptado el 19 de abril de 2016.

Correspondencia a:

Dr. Carlos Benítez G. Departamento de Gastroenterología, Escuela de Medicina, Pontificia Universidad Católica de Chile. Marcoleta 367, 833-0024, Santiago, Chile. Teléfono: $+56-2-23543820$

Fax: +56-2-26397780 benitezc@gmail.com

\section{Aumento aislado y sostenido de aspartato aminotransferasa por presencia de macroenzimas. Caso clínico}

\author{
VERÓNICA BUSTAMANTE ${ }^{1}$, JUAN PABLO ARAB ${ }^{2}$, \\ FLORENCIA TERC ${ }^{2}$, HELENA POGGI ${ }^{1, \mathrm{a}}$, MANUELA GOYCOOLEA ${ }^{1, \mathrm{a}}$, \\ MARCO ARRESE ${ }^{2}$, TERESITA QUIROGA ${ }^{1}$, CARLOS BENÍTEZ $^{2}$
}

\section{Persistent elevation of aspartate aminotransferase (AST) due to the presence of macro-AST. Report of one case}

We report an asymptomatic 23-year-old woman with an isolated and persistent increase in serum levels of aspartate aminotransferase (AST). An extensive work up including laboratory and image testing revealed no abnormalities thus suggesting the presence of macro-AST. A polyethylene glycol (PEG) precipitation assay was performed and confirmed the presence of macro-AST.

(Rev Med Chile 2016; 144: 1078-1082)

Key words: Aspartate Aminotransferases; Enzymes; Polyethylene Glycols.
L a aspartato aminotransferasa (AST) es una enzima ampliamente utilizada en el estudio de las enfermedades hepáticas ${ }^{1}$. No obstante, se encuentra presente en células de diversos tejidos, tales como el músculo esquelético y cardíaco, riñón, eritrocitos y hepatocitos, entre otros. Así, si bien su elevación está generalmente relacionada a hepatopatías, el diagnóstico diferencial de esta alteración de laboratorio debe incluir consideraciones respecto a patología muscular, cardiaca y endocrina ${ }^{2,3}$. Una causa infrecuente de la elevación aislada de AST es la existencia de macroenzimas circulantes ${ }^{4}$. Estas corresponden a complejos de alto peso molecular formados por múltiples moléculas enzimáticas unidas por inmunoglobulinas, lo cual reduce su aclaramiento. La presencia de macro-AST es infrecuentemente considerada en la práctica clínica. A continuación, a propósito de la descripción de un caso clínico, se revisan conceptos actuales sobre la macro-AST y sus implicancias clínicas.

\section{Caso clínico}

Mujer de 23 años, con antecedentes de síndrome de ovario poliquístico, derivada por elevación de aminotransferasas. Dentro de sus antecedentes se consignó que era usuaria de anticonceptivos orales, no había sido sometida a cirugías, no fumaba, bebedora de alcohol muy ocasionalmente. Su padre era portador de un síndrome de Gilbert y su tío falleció por un mieloma múltiple.

En un control general se evaluaron las pruebas hepáticas: AST $224 \mathrm{UI} / \mathrm{L}(\mathrm{VN}<25)$, alanino aminotransferasa (ALT) $13 \mathrm{UI} / \mathrm{L}(\mathrm{VN}<30)$, gammaglutamiltransferasa (GGT) $20 \mathrm{UI} / \mathrm{L}$ (VN $<50$ ), fosfatasas alcalinas (FA) $46 \mathrm{UI} / \mathrm{L}(\mathrm{VN}<$ 100 ), bilirrubina total $0,37 \mathrm{mg} / \mathrm{dL}$ (VN 0-1), bilirrubina directa $0,12 \mathrm{mg} / \mathrm{dL}(\mathrm{VN}<0,3)$, INR 1,1 . Un mes más tarde nuevamente la AST estaba elevada (261 UI/L), siendo el resto de las pruebas hepáticas del todo normales. Dado que la paciente se encontraba asintomática, se decidió 
repetir los exámenes dos meses después, persistiendo la elevación aislada de la AST (293 UI/L). A raíz de esto, se realizó un extenso estudio para descartar hepatopatías: hepatitis virales y enfermedades hepáticas autoinmunes, metabólicas (determinación de niveles de alfa-1 antitripsina y perfil de hierro) y enfermedad celiaca mediante determinaciones de anticuerpos anti-endomisio. Todos los resultados se encontraron dentro de los intervalos de referencia. La ecografía abdominal fue también normal.

Puesto que tras 6 años de seguimiento la paciente permaneció asintomática se planteó la posibilidad de estar en presencia de una macroenzima. Para comprobarlo se decidió utilizar el método de polietilenglicol (PEG), que bajo las condiciones apropiadas tiene la propiedad de precipitar los complejos de macroenzimas y permite medir la actividad remanente en el sobrenadante. Para ello se analizó una nueva muestra de suero de la paciente, cuya actividad de AST determinada en el multianalizador Modular ${ }^{\circledR}$ (Roche/Hitachi) fue de 176,5 UI/L. En esta muestra se realizó una precipitación con PEG según el protocolo descrito por Olukaga et al. ${ }^{5}$, en el cual se mezcla el suero de la paciente con una solución acuosa de PEG 6.000 al $25 \%$ en una relación 1:2 y posteriormente se centrifuga para precipitar los complejos. El procedimiento se realizó en triplicado y se procesaron como control muestras de suero de 5 pacientes con elevación de AST no aislada. Los resultados se pueden observar en la Tabla 1. Las muestras de la paciente mostraron un porcentaje de actividad post precipitación con PEG (\%PPA, PEG precipitation activity) elevado en todos los tubos del ensayo: 98,0\%, 98,8\% y 97,7\%. En los pacientes controles, en cambio, los porcentajes fueron menores, obteniéndose en promedio un \%PPA de $55,5 \%$, con un intervalo entre $31,6 \%$ y $64,0 \%$. A diferencia de lo que se observó en los controles, casi la totalidad de la actividad enzimática de la paciente en estudio se encontró en el precipitado, lo que sugirió la presencia de una macroenzima.

\section{Discusión}

A diferencia de la ALT, la AST tiene una baja especificidad y puede originarse en distintos reservorios celulares incluyendo células hepáticas, cardiacas, renales, musculares y glóbulos rojos ${ }^{1,6}$. La función de AST y ALT consiste en transferir grupos amino desde una $\alpha$-aminoácido a un $\alpha$-cetoácido, durante el ciclo del ácido cítrico ${ }^{6}$. Ambas enzimas son liberadas desde el citoplasma a la circulación cuando hay daño o muerte celular secundario a diferentes noxas (hepatitis virales, enfermedades autoinmunes, tóxicos, alcohol, entre otras) $)^{2,3,6,7}$. Exceptuando las hepatitis alcohólicas, prácticamente en todas las hepatopatías se elevan predominantemente los niveles de ALT, dado que esta enzima tiene una vida media mayor $(50 \mathrm{~h} \text { versus } 16 \mathrm{~h} \text {, respectivamente })^{3,7}$. Existen una serie de condiciones que elevan la AST, estas incluyen la hemólisis por efecto de la punción o por el trasporte de la muestra ${ }^{7}$. En la Figura 1 se

Tabla 1. Niveles de AST en el suero, en el sobrenadante post precipitación con PEG y \%PPA (porcentaje actividad post-precipitación con PEG) del paciente y los controles.

Se observa un alto \%PPA en todos los triplicados de la paciente ( $>73 \%$ ), lo que indica presencia de macroenzimas. Los 5 pacientes usados como controles negativos tuvieron un bajo \%PPA

\begin{tabular}{|lccc|}
\hline & AST en suero (UI/L) & $\begin{array}{c}\text { AST en sobrenadante } \\
\text { post precipitación (UI/L) }\end{array}$ & \%PPA \\
\hline Paciente & 176,5 & 3,6 & 98,0 \\
& 176,5 & 2,0 & 98,9 \\
\hline Control 1 & 176,5 & 4,0 & 97,7 \\
\hline Control 2 & 195,5 & 72,6 & 62,9 \\
\hline Control 3 & 350,4 & 156,6 & 55,3 \\
\hline Control 4 & 116,7 & 42,0 & 64,0 \\
\hline Control 5 & 264,4 & 96,0 & 63,7 \\
\hline
\end{tabular}




\section{Elevación aislada de la aspartato-aminotransferasa (AST)}

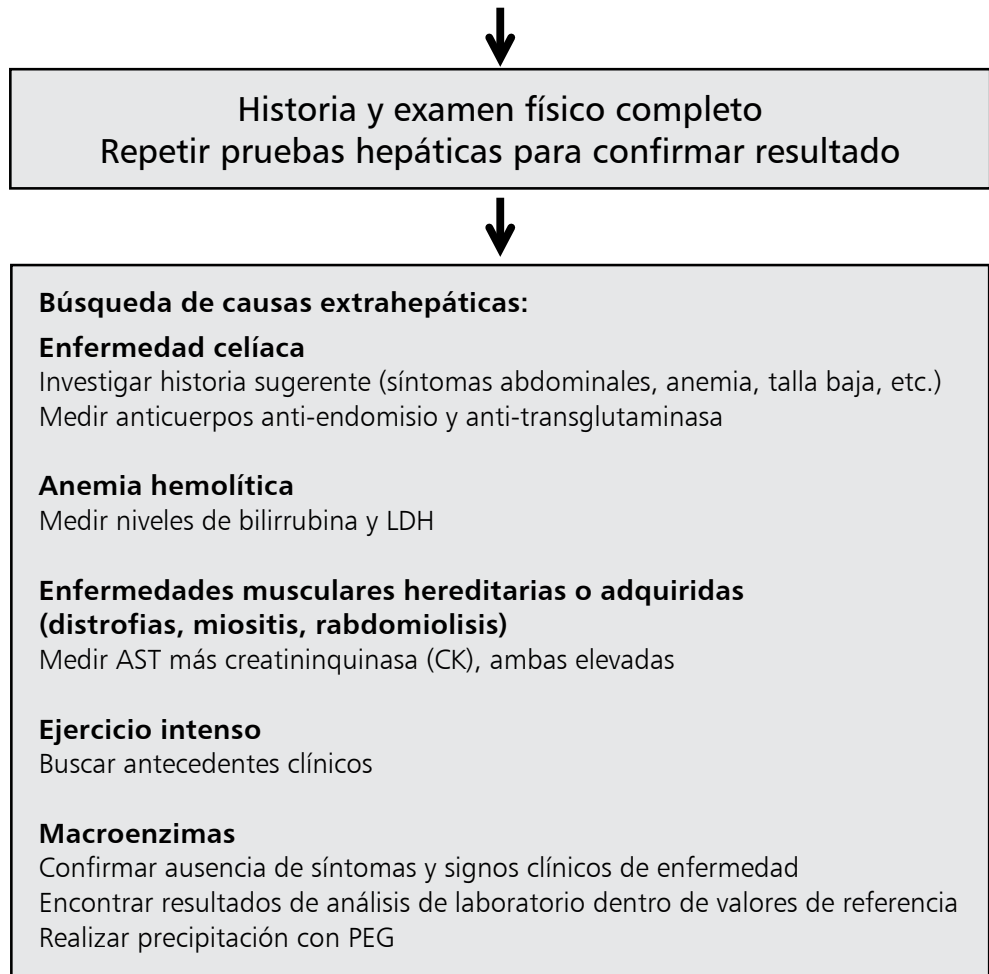

Figura 1. Algoritmo de orientación al diagnóstico frente a elevación de aminotransferasas.

presenta un algoritmo para la evaluación de la elevación aislada de la AST, el cual fue empleado en la paciente aquí presentada ${ }^{4,6-8}$.

Las macroenzimas fueron descritas por primera vez en 1964 como complejos de alto peso molecular formados por moléculas enzimáticas unidas entre sí formando polímeros, o unidos a componentes del plasma de alto peso molecular como inmunoglobulinas o proteínas ${ }^{9,10}$. Por lo anterior, su aclaramiento renal se encuentra marcadamente reducido sin que exista un aumento de su liberación desde las células ${ }^{6,9,11}$. La causa por la que se forman estos complejos aún no se ha dilucidado, pero se postula una desregulación de la tolerancia inmune que llevaría a la unión de las inmunoglobulinas, teoría que surgió por una asociación con patologías autoinmunes ${ }^{6,10,11}$. También existe asociación con otras condiciones como distintos tipos de cáncer.
Existen pocos estudios de la prevalencia de macroenzimas, pero los datos que existen indican que varía según el tipo de enzima y la patología de base. Un estudio estimó que la macroamilasemia tendría una prevalencia de $0,98 \%$ a $2,58 \%$ en población general, mientras que la de LDH ocurriría en $<1 / 10.000$ sujetos $^{6,12}$. En la actualidad no existe evidencia que indique que las distintas macroenzimas produzcan alguna enfermedad, sino que corresponderían, en algunos casos, a una manifestación de una patología de base ${ }^{13-16}$. La prevalencia de macro-AST en la población general se desconoce, podría ser menor que la de otras macroenzimas ${ }^{9,17}$. En un estudio de 44 niños sanos, con elevación persistente y aislada de AST, se encontraron complejos de macroenzimas en un tercio de los $\operatorname{casos}^{11}$. Además, su presencia parece ser significativamente mayor en enfermedades hepáticas que en sujetos sanos. Por ejemplo, se 
encontró presencia de macro-AST en $90 \%$ de los pacientes con carcinoma hepatocelular, $41,8 \%$ de pacientes con hepatitis crónica, $62 \%$ de pacientes con cirrosis y en $66 \%$ de las hepatitis alcohólicas ${ }^{6,18}$.

Los macro-complejos enzimáticos se pueden medir con distintas técnicas. La precipitación con PEG utilizada en el presente caso es sencilla, y se usa para el diagnóstico de diferentes macroenzimas $^{19}$. El uso del PEG en determinadas concentraciones tiene como efecto la sustracción de los solventes, y por ello la precipitación de proteínas como inmunoglobulinas y los complejos formados por ellas ${ }^{19,20}$, midiéndose así la actividad antes de la precipitación y la actividad remanente en el sobrenadante. Los resultados se pueden expresar como actividad remanente en el sobrenadante (\% recuperación) o como actividad post precipitación con PEG (\%PPA $)^{19}$. En distintas publicaciones se considera diagnóstica una disminución de $73 \%$ o más en la actividad en el sobrenadante (\%PPA $)^{21}$. En el caso de la paciente aquí descrita, el \%PPA está muy por sobre $73 \%$, lo que confirma la sospecha de la presencia de una macroenzima. Otros autores sugieren el uso de 2 puntos de corte $(67,1$ y $82,2 \%)$ lo que estable un rango en el cual el diagnóstico es incierto ${ }^{14,19}$. En nuestro caso, todos los sueros utilizados como controles se obtuvo un $\%$ PPA muy por debajo de los límites propuestos, por lo que no hubo resultados de difícil interpretación. Otra limitación de este estudio es que los valores de referencia usados fueron establecidos en base a estudios en pacientes con sospecha de macroenzimas y no en sujetos normales, lo que puede entregar resultados sesgados ${ }^{19,21}$. No obstante, la precipitación con PEG es de gran utilidad en la medida que se reconozcan sus limitaciones.

En resumen, frente a un caso de una elevación aislada y persistente de AST, en ausencia de enfermedad aparente, debe sospecharse la presencia de macroenzimas, lo cual evita estudios y costos innecesarios.

\section{Referencias}

1. Lott JA, Wolf PL. Alanine and aspartate aminotransferase (ALT and AST). In: Lott JA, Wolf PL eds. Clinical enzymology: a case-oriented approach. New York: Field, Rich, and Associates 1986; 111-38.

2. Giannini EG, Testa R, Savarino V. Liver enzyme alteration: a guide for clinicians. CMAJ 2005; 172: 367-79.

3. Pratt DS, Kaplan MM. Evaluation of abnormal liver-en- zyme results in asymptomatic patients. $\mathrm{N}$ Engl J Med 2000; 342: 1266-71.

4. Lee M, Vajro P, Keeffe EB. Isolated aspartate aminotransferase elevation: think macro-AST. Dig Dis Sci 2011; 56: 311-3.

5. Olukoga AO, Kane JW. Macroprolactinaemia: validation and application of the polyethylene glycol precipitation test and clinical characterization of the condition. Clin Endocrinol (Oxf) 1999; 51: 119-26.

6. Krishnamurthy S, Korenblat KM, Scott MG. Persistent increase in aspartate aminotransferase in an asymptomatic patient. Clin Chem 2009; 55: 1573-5.

7. Biochemistry TNAoC: Laboratory Guidelines for Screening, Diagnosis and Monitoring of Hepatic Injury. In: Laboratory Medicine Practice Guidelines. Volume 12. Washington, DC: National Academy of Clinical Biochemistry, 2000; 61.

8. Wener RR, Loupatty FJ, Schouten WE. Isolated elevated aspartate aminotransferase: a surprising outcome for clinicians. Neth J Med 2012; 70: 136-8.

9. Chtioui H, Mauerhofer O, Gunther B, Dufour JF. Macro-AST in an asymptomatic young patient. Ann Hepatol 2010; 9: 93-5.

10. Plebani M, Wu AH. Circulating macrocomplexes: old wine in new bottles? Clin Chem Lab Med 2011; 49: 75960.

11. Turecky L. Macroenzymes and their clinical significance. Bratisl Lek Listy 2004; 105: 260-3.

12. Onuigbo M, Yin R. Macro-aspartate transaminasemia: the macroenzyme phenomenon as a diagnostic dilemma. Mayo Clin Proc 2000; 75: 1223-4.

13. Orlando R, Carbone A, Lirussi F. Macro-aspartate aminotransferase (macro-AST). A 12-year follow-up study in a young female. Eur J Gastroenterol Hepatol 2003; 15: 1371-3.

14. Caropreso M, Fortunato G, Lenta S, et al. Prevalence and long-term course of macro-aspartate aminotransferase in children. J Pediatr 2009; 154: 744-8.

15. Fortunato G, Iorio R, Esposito P, Lofrano MM, Vegnente A, Vajro P. Macroenzyme investigation and monitoring in children with persistent increase of aspartate aminotransferase of unexplained origin. J Pediatr 1998; 133: 286-9.

16. Lord R, Fahie-Wilson M, Suri S. A paediatric case of macro aspartate aminotransferase. Ann Clin Biochem 2008; 45: 323-4.

17. Konttinen A, Murros J, Ojala K, Salaspuro M, Somer $\mathrm{H}$, Rasanen J. A new cause of increased serum aspartate aminotransferase activity. Clin Chim Acta 1978; 84: 1457.

18. Tameda M, Shiraki K, Ooi K, et al. Aspartate amino- 
transferase-immunoglobulin complexes in patients with chronic liver disease. World J Gastroenterol 2005; 11 : 1529-31.

19. Patteet L, Simoens M, Piqueur M, Wauters A. Laboratory detection of macro-aspartate aminotransferase: case report and evaluation of the PEG-precipitation method. Clin Biochem 2012; 45: 691-3.
20. Fahie-Wilson M, Halsall D. Polyethylene glycol precipitation: proceed with care. Ann Clin Biochem 2008; 45: 233-5.

21. Wyness SP, Hunsaker JJ, La'ulu SL, Roberts WL. Reference intervals for six enzymes after polyethylene glycol precipitation and ultrafiltration. Clin Chim Acta 2011; 412: 1161-2. 